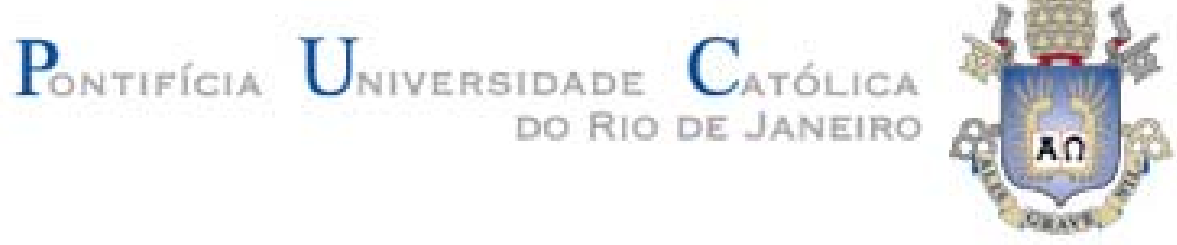

Leonardo Soares da Silva

Desmistificação do Luxo: Um Estudo Sobre as Motivações de Compra de Réplicas

Dissertação de Mestrado

Dissertação apresentada ao Programa de Pósgraduação em Administração de Empresas da PUCRio como requisito parcial para obtenção do titulo de Mestre em Administração de Empresas.

Orientador: Prof. Luís Alexandre Grubits de Paula Pessôa

Rio de Janeiro

Setembro 2012 
Leonardo Soares da Silva

\section{Desmistificação do Luxo: Um Estudo Sobre as Motivações de Compra de Réplicas}

Dissertação apresentada como requisito parcial para obtenção do grau de Mestre pelo Programa de Pósgraduação em Administração de Empresas da PUC-Rio. Aprovada pela Comissão Examinadora abaixo assinada.

Prof. Luís Alexandre Grubits de Paula Pessôa Orientador Departamento de Administração - PUC-Rio

Prof. Luis Fernando Hor-Meylll Alvares Departamento de Administração - PUC-Rio

Prof. Eduardo André Teixeira Ayrosa Fundação Getúlio Vergas

Prof ${ }^{a}$. Mônica Herz

Vice-Decana de Pós-Graduação do CCS

Rio de Janeiro, 17 de setembro de 2012 
Todos os direitos reservados. É proibida a reprodução total ou parcial do trabalho sem autorização da universidade, do autor e do orientador.

\section{Leonardo Soares da Silva}

Graduou-se em Administração de Empresas na UFRJ (Universidade Federal do Rio de Janeiro) em 2008.Trabalha atualmente na empresa CAIXA Econômica Federal, na cidade do Rio de Janeiro.

Ficha Catalográfica

Silva, Leonardo Soares da

Desmistificação do luxo : um estudo sobre as motivações de compra de réplicas / Leonardo Soares da Silva ; orientador: Luís Alexandre Grubits de Paula Pessôa. - 2012.

$138 \mathrm{f.} ; 30 \mathrm{~cm}$

Dissertação (mestrado)-Pontifícia Universidade Católica do Rio de Janeiro, Departamento de Administração, 2012.

Inclui bibliografia

1. Administração - Teses. 2. Falsificação. 3. Luxo. 4. Valores. 5. Cadeia meios-fim. 6. Laddering. I. Pessôa, Luís Alexandre Grubits de Paula. II. Pontifícia Universidade Católica do Rio de Janeiro. Departamento de Administração. III. Título.

CDD:658 
Dedico esse trabalho aos queridos professores Eduardo André Teixeira Ayrosa, Luís Alexandre Grubits de Paula Pessôa e Luís Fernando Hor-Meyll Alvares por compartilhar comigo sua experiência e seus conhecimentos no início de minha jornada acadêmica. 


\section{Agradecimentos}

A Deus por ter me agraciado com esta vida repleta de momentos felizes. Obrigado por todas as graças que me foram concedidas.

A minha família, razão de todo o meu viver e o principal motivo de meus sorrisos. Obrigado a meu pai, Cinésio Souza da Silva, e a minha mãe, Rita de Cássia Soares da Silva, pelo amor, pelo carinho, pelo apoio e força que sempre me deram. Vocês são o exemplo que eu sigo. Obrigado a minha irmã Lívia Soares da Silva, pelo amor e carinho que seu coração sempre reservou para mim. Amo todos vocês.

Ao meu orientador Luís Alexandre Grubits de Paula Pessôa pelos ensinamentos, pelas reflexões, pelo apoio, pelo compromisso e pelo rigor com que me guiou nesta etapa tão importante de minha vida. Obrigado por ampliar minha paixão pelo marketing e minha vontade de ingressar na vida acadêmica.

À Renata Couto pela fundamental participação e contribuição na realização das entrevistas. Muito obrigado pela sua amizade e parceria, pelo apoio e torcida nesse momento tão importante para mim.

Às 15 mulheres entrevistadas que gentilmente aceitaram participar desta pesquisa, contribuindo com suas experiências para que essa etapa fundamental fosse concretizada.

Ao professor Luís Fernando Hor-Meyll por compartilhar seus conhecimentos do método utilizado neste trabalho.

A Daniele Vasconcelos, Fabrícia Peixoto, Jeane Lucena, Luciene Lima, Marcus Vinicius Silva de Miranda, Priscilla Farias, Reinaldo Brás, Roberto Candreva, Rodolfo Lima, Thereza Lamoglia, Vanessa Candreva, Valesca Cunha e Waleska Vieira por ouvir meus desabafos, minhas angústias, por torcerem por meu sucesso e por tornar essa caminhada mais leve com seus sorrisos, brincadeiras e principalmente com a amizade com que me agraciaram.

Ao Professor Paulo Cesar Lopes Pereira por ter me apresentado o Marketing e, a partir de sua competência como mestre, ter me inspirado a ingressar na vida acadêmica. 


\section{Resumo}

Silva, Leonardo Soares da; Pêssoa, Luís Alexandre Grubits de Paula. Desmistificação do Luxo: Um Estudo Sobre as Motivações de Compra de Réplicas. Rio de Janeiro, 2012. 138p. Dissertação de Mestrado Departamento de Administração de Empresas, Pontifícia Universidade Católica do Rio de Janeiro.

Seguindo uma tendência mundial, o consumo de produtos falsificados tem se ampliado consideravelmente no Brasil, com relevantes consequências econômicas e sociais. Apesar de sua característica de exclusividade, os produtos de marcas de luxo não são imunes a esse processo. Réplicas de artigos como bolsas e relógios de marcas historicamente inacessíveis para muitos, por exemplo, podem ser adquiridos com facilidade no país. A desmistificação do luxo é, em grande parte, resultado da evolução do seu significado: partindo de uma lógica artesanal para uma visão financeira e industrial, o luxo caminhou pela modernidade e pós-modernidade - era do consumo movido pelo simbolismo e pela união do real e do imaginário, até, finalmente, democratizar-se através do “novo luxo”, abrindo as portas para a falsificação. Nesse contexto, o presente estudo buscou responder à seguinte pergunta: Quais valores podem ser considerados responsáveis pela decisão de compra de bolsas falsificadas da marca Louis Vuitton? Para tanto, foi realizada uma pesquisa exploratória, em que 15 mulheres que compraram bolsas Louis Vuitton falsificadas foram submetidas a entrevistas em profundidade analisadas pela técnica Laddering. O resultado do estudo sugere que, para além dos atributos do produto, avaliado como de elevada beleza, a bolsa faz com que as mulheres sintam-se possuidoras de imponência e status. Ao comprarem a bolsa Louis Vuitton, mesmo falsificada, as consumidoras buscam o pertencimento e procuram elevar a autoestima, valor que atua como principal motivador da decisão de compra.

\section{Palavras-chave}

Falsificação; luxo; valores; cadeia meios-fim; laddering 


\section{Abstract}

Silva, Leonardo Soares da; Pêssoa, Luís Alexandre Grubits de Paula (Advisor). Demystification of Luxury: A Study about the Motivations of Buying Product Replicas. Rio de Janeiro, 2012. 138p. MSc. Dissertation Departamento de Administração de Empresas, Pontifícia Universidade Católica do Rio de Janeiro.

Following a global trend, the consumption of counterfeit goods is substantially spreading in Brazil, with significant economic and social consequences. Despite its uniqueness, luxury brand products are not immune to this process. Replicas of items such as handbags and watches produced by brands that are historically inaccessible for many, for example, can be easily purchased in the country. The demystification of luxury is the result of the evolution of its meaning: starting from craft logic onto a financial and industrial vision, luxury strode through modernity and post-modernity - the consumption era driven by symbolism and the merging of real and imaginary until finally democratize itself through what is known as "new luxury", making counterfeit possible. In this context, the present study intends to comply with the following question: Which values could be held responsible for the buying decision of counterfeit Louis Vuitton handbags? To this end, we conducted an exploratory study in which 15 female subjects who bought counterfeit Louis Vuitton handbags were subjected to in-depth interviews analyzed using Laddering. The result of the study suggests that besides the product attributes, evaluated as extremely beautiful, to own a handbag made women feel imponent and with a certain status. When acquiring a Louis Vuitton hanbag, even though counterfeit, female consumers crave for belonging and raising their self-esteem, a value that is the primary motivation of the purchase decision.

\section{Keywords}

Counterfeiting; luxury; values; means-end chain; laddering. 


\section{Sumário}

1. Introdução 12

1.1. Contextualização do Problema 14

1.2. O Problema de Pesquisa 16

1.3. Objetivos da Pesquisa 16

$\begin{array}{ll}\text { 1.3.1. Objetivo da Pesquisa } & 16\end{array}$

1.3.2. Objetivos Intermediários 16

1.4. Relevânica 16

1.5. Delimitação 18

1.6. Organização do Trabalho 18

2. Referencial Teórico 21

2.1. Luxo 21

2.1.1. Conceito 21

2.1.2. A Evolução do Luxo: Do Sagrado ao Banal 23

2.2. Falsificação $\quad 27$

2.2.1. Definição $\quad 27$

2.2.2. O Mercado de Falsificados 31

2.2.3. O Consumo de Falsificados 33

2.2.4. O Perfil do Consumidor de Falsificados 35

2.3. A Cadeia Meios Fim 38

2.3.1. Atributos 40

2.3.2. Consequências 41

2.3.3. Valores 42

2.3.3.1. Valores - A Abordagem de Rokeach 44

2.3.3.2. Valores - A Abordagem de Jean - Marie Floch 54

3. Métodos 57

3.1. A Seleção da Amostra

3.2. O Método de Coleta dos Dados: O Laddering 59

3.2.1. A Coleta de Dados: As Entrevistas 60

3.2.2. A Análise e Interpretação de Resultados 64

3.2.2.1. A Análise de Conteúdo 64

3.2.2.2. Matriz de Implicação 65

3.2.2.3. Mapa Hierárquico de Valor 66

3.2.2.4. Orientações de Percepção Dominantes 68

3.2.3. Limitações do Laddering 70 
4. Análise $\quad 72$

4.1. Análise de Conteúdo $\quad 72$

4.2. Matriz de Implicação

4.3. Mapa Hierárquico de Valor $\quad 77$

4.4. Orientações de Percepção Dominantes 81

5. Análise e Discussão dos Resultados 83

5.1. Análise dos Resultados 83

5.1.1. O Valor Terminal $\quad 84$

5.1.2. Consequências 88

5.1.3. Atributos 90

5.2. Análise dos Resultados - Calores Sob a Ótica de Jean-Marie
Floch

5.3. O Luxo e a Falsificação 97

6. Considerações Finais 100

7. Referências Bibliográficas 104

$\begin{array}{ll}\text { 8. Anexos } & 111\end{array}$ 


\section{Lista de tabelas}

Tabela 1 - Escala de Valores de Rokeach 53

Tabela 2 - Perfil das entrevistadas $\quad 58$

Tabela 3 - Sumário de Códigos $\quad 73$

Tabela 4 - Atributos Concretos e Abstratos $\quad 74$

Tabela 5 - Consequências Funcionais e Psicossociais 75

Tabela 6 - Valores Instrumentais e Terminais 76

Tabela 7 - Resumo das Relações Diretas e Indiretas de Cada
Elemento

Tabela 8 - Orientações de Percepção Dominante 82 


\section{Lista de figuras}

Figura 1 - Os valores de consumo de Floch 55

Figura 2 - Mapa Hierárquico de Valor

78

Figura 3 - Valores de consumo aplicados à Louis Vuitton

94 